\title{
Epithelial to Mesenchymal Transition Expression Profiles as Predictive Biomarkers of Recurrence Following Resection of HCC: Implications for Current Clinical Use and Future Stratification for Systemic Therapy
}

\author{
Adam C. Yopp ${ }^{1}$ and Amit G. Singal ${ }^{2}$ \\ ${ }^{1}$ Division of Surgical Oncology, Department of Surgery, University of Texas Southwestern Medical Center, Dallas, TX; \\ ${ }^{2}$ Division of Digestive and Liver Diseases, Department of Medicine, University of Texas Southwestern Medical Center, \\ Dallas, TX
}

Hepatocellular carcinoma (HCC) is the fifth most common cancer worldwide and is responsible for more than 500,000 deaths annually. ${ }^{1}$ Hepatic resection, liver transplantation, or tumor ablation are the only curative options for HCC. Despite surgical resection being the preferred treatment modality for small, solitary lesions in patients with preserved liver function, postoperative recurrence remains high, and is a major determinant of overall outcome. The overall rate of HCC recurrence is more than $50 \%$ in most published reports with the vast majority of recurrences occurring in the remnant liver. Intrahepatic recurrences are time dependent and thought to occur secondary to either metastasis from the initial resected lesion (within 2 years of resection) or de novo tumors arising from a field defect of the underlying liver parenchyma (beyond 2 years following resection). ${ }^{2}$ The importance of distinguishing between the two mechanisms is important for future adjuvant systemic therapy trials but can be difficult to discern outside of routine clinicopathological tumor features. Early tumor recurrences are generally seen in tumors with poor pathological features, including poorly differentiated tumors, vascular invasion, and/or multifocality. ${ }^{3}$

Epithelial mesenchymal transition (EMT) is an important event in tumor metastases whereby epithelial cell layers lose polarity and cell-cell contacts, thus allowing

(C) Society of Surgical Oncology 2014

First Received: 29 April 2014;

Published Online: 30 July 2014

A. C. Yopp

e-mail: adam.yopp@utsouthwestern.edu detachment and increasing cell motility. A hallmark of EMT is the loss of the cell adhesion molecule E-cadherin and the induction of the transcription factors: Twist, Snail 1, Snail 2, ZEB 1, and ZEB 2.,5

In the current study, Yamada et al. report on the use of factors associated with EMT as a predictive biomarker for recurrence following resection of HCC. ${ }^{6}$ The authors identified a mesenchymal group by the loss of E-cadherin as being associated with a significantly worse disease-free survival (DFS) but no change in overall survival compared with a group defined as epithelial with preserved E-cadherin. This finding is similar to previously published studies demonstrating the presence of E-cadherin as a prognostic biomarker of DFS. ${ }^{4,5,7}$ However, the absence of other clinicopathological factors in the mesenchymal group, including vascular invasion, histological differentiation, and infiltrative tumor growth, previously demonstrated to be associated with early recurrence is surprising and may be a reflection of the relative late nature of tumor recurrence in both the epithelial and mesenchymal groups. ${ }^{3}$ Although there is a significant difference in DFS between the mesenchymal and epithelial groups at 5 years, the split of the Kaplan-Meier curves seems to be more pronounced 2 years following resection, indicating a high likelihood of the formation of de novo tumors and not intrahepatic metastasis as postulated by the authors. Unfortunately, the only way to discern between intrahepatic metastasis and de novo tumors is to test clonality of the tumors, a process that is cumbersome and not clinically applicable. $^{2}$

The high recurrence rate following surgical resection of HCC has led to the attempt of identifying biomarkers that 
stratify resected patients into more and less favorable subgroups potentially aiding in better treatment decisions. Although a novel concept, stratification of HCC patients with early-stage disease by tumor characteristics, whether molecular or genetic markers, is not without potential issues. Even if an externally validated biomarker is developed, similar to recent genome-wide expression profiles, stratifying resected $\mathrm{HCC}$ patients into recurrence patterns, those patients with an "unfavorable" biomarker or profile would still best be offered surgical resection rather than locoregional or systemic therapy due to significantly better overall outcomes. ${ }^{8,9}$ Until more efficacious systemic therapies are developed in an adjuvant setting to prevent early intrahepatic recurrences presumably secondary to tumor metastasis and not from a "field defect" of cirrhotic liver, prognostic biomarkers of surgical specimens should be limited to discerning between offering surgical resection or liver transplantation to applicable patients.

The authors shed some light on stratification of patients who would receive potential benefit from systemic therapy following resection by demonstrating that epithelial cell lines were more sensitive to sorafenib than their mesenchymal counterparts. This is an important albeit not surprising observation given the favorable tumor biology of the epithelial differentiation. Much more exciting was their finding that blocking IL-6, a tumor cytokine prognostic of poor outcome in HCC, in mesenchymal cell lines increased sorafenib sensitivity significantly. It will be interesting to see if future animal models with mesenchymal HCC tumor xenografts respond more favorably to combination IL-6 blockade/sorafenib than with sorafenib alone. Given the lack of efficacious adjuvant treatment strategies after hepatic resection for HCC, this is a promising start and the authors should be congratulated.

Potentially the most interesting aspect of EMT expression profiles as biomarkers is their role in stratifying patients not eligible for curative therapies. Unfortunately, worldwide less than $25 \%$ of patients presenting with HCC are eligible for liver transplantation, surgical resection, or ablative techniques due to decompensated liver or performance status or advanced stage of tumor. It is in these noncurative situations where stratification of patients to the most efficacious therapy would be most valuable. This study like others before it does not attempt to characterize their biomarker in a nonsurgical patient, likely due to an unavailability of tissue. It is unclear whether biomarkers developed and externally validated in surgical resection specimens correlate to advanced stage HCC. Due to the shift in diagnosis of HCC through radiological and not pathological means as advocated by group consensus, it is unlikely whether we will ever be able to confidently stratify advanced HCC patients to appropriate treatment strategies. ${ }^{10}$

In conclusion, the loss of E-cadherin as seen in mesenchymal differentiation of HCC resected patients is associated with increased rate of recurrence at 5 years. Increase in EMT transcription factors are seen in the mesenchymal phenotype and also are predictive of worse outcome. Tumors with mesenchymal differentiation are less sensitive to sorafenib, but with IL-6 blockade these tumors regain sensitivity, which has important implications on the potential use of adjuvant systemic therapy.

\section{REFERENCES}

1. Parkin DM, Bray F, Ferlay J, et al. Estimating the world cancer burden: Globocan 2000. Int J Cancer. 2001;94:153-6.

2. Ng IO, Guan XY, Poon RT, et al. Determination of the molecular relationship between multiple tumour nodules in hepatocellular carcinoma differentiates multicentric origin from intrahepatic metastasis. J Pathol. 2003;199:345-53.

3. Poon RT, Fan ST, Ng IO, et al. Different risk factors and prognosis for early and late intrahepatic recurrence after resection of hepatocellular carcinoma. Cancer. 2000;89:500-7.

4. Thiery JP. Epithelial-mesenchymal transitions in tumour progression. Nat Rev Cancer. 2002;2:442-54.

5. Lee TK, Poon RT, Yuen AP, et al. Twist overexpression correlates with hepatocellular carcinoma metastasis through induction of epithelial-mesenchymal transition. Clin Cancer Res. 2006;12:5369-76.

6. Yamada S, Okumura N, Wei L, et al. Epithelial to mesenchymal transition is associated with shorter disease-free survival in hepatocellular carcinoma. Ann Surg Oncol. doi:10.1245/s10434014-3779-2.

7. Zhou YM, Cao L, Li B, et al. (2012) Clinicopathological significance of ZEB1 protein in patients with hepatocellular carcinoma. Ann Surg Oncol. 19:1700-6.

8. Nault JC, De Reynies A, Villanueva A, et al. (2013) A hepatocellular carcinoma 5-gene score associated with survival of patients after liver resection. Gastroenterology. 145:176-87.

9. Villanueva A, Hoshida Y, Battiston C, et al. (2011) Combining clinical, pathology, and gene expression data to predict recurrence of hepatocellular carcinoma. Gastroenterology. 140:150 $12 \mathrm{e} 2$.

10. Bruix J, Sherman M. (2011) Management of hepatocellular carcinoma: an update. Hepatology. 53:1020-2. 